\begin{tabular}{|c|l|}
\hline Title & $\begin{array}{l}\text { Negative initial phase shift of Kerr rotation generated from the building-up process of resident electron spin polarization } \\
\text { in aCdTe single quantum well }\end{array}$ \\
\hline Author(s) & Y an, L. -P.; Kurosawa, M.; Kaji, R.; Karczewski, G.; Takey ama, S.; A dachi, S. \\
\hline Citation & $\begin{array}{l}\text { Physical Review B, 90(20), 205307 } \\
\text { https://doi.org/10.1103/PhysRevB.90.205307 }\end{array}$ \\
\hline Issue Date & 201411-15 \\
\hline Doc URL & http://hdl.handle.net/2115/57749 \\
\hline Rights & @2014 A merican Physical Society \\
\hline Type & article \\
\hline File Information & PhysRevB.90.205307.pdf \\
\hline
\end{tabular}

Instructions for use 


\title{
Negative initial phase shift of Kerr rotation generated from the building-up process of resident electron spin polarization in a CdTe single quantum well
}

\author{
L.-P. Yan, ${ }^{1}$ M. Kurosawa, ${ }^{1}$ R. Kaji, ${ }^{1}$ G. Karczewski ${ }^{2}$ S. Takeyama,${ }^{3}$ and S. Adachi ${ }^{1, *}$ \\ ${ }^{1}$ Department of Applied Physics, Hokkaido University, N13 W8, Kitaku, Sapporo 060-8628, Japan \\ ${ }^{2}$ Institute of Physics, Polish Academy of Sciences, 02-668 Warsaw, Poland \\ ${ }^{3}$ Institute for Solid State Physics, University of Tokyo, 277-8581 Kashiwa, Japan
}

(Received 17 August 2014; revised manuscript received 14 October 2014; published 19 November 2014)

\begin{abstract}
Initial phase shift in a precessional motion of resident electron-spin polarization is studied in a $\mathrm{CdTe} / \mathrm{Cd}_{0.85} \mathrm{Mg}_{0.15} \mathrm{Te}$ single quantum well using a time-resolved Kerr rotation technique. The generation dynamics of resident electron-spin polarization involve the formation and transformation of the associated optically excited states and are complicated particularly in the early time region. A careful analysis of the phase shift gives a deep understanding of the generation processes. In the experiments, the negative phase shift of the resident electron-spin polarization is observed, and the mechanism associated especially with a quick hole spin flip in negative trions is studied through the dependences on excitation power and magnetic field strength.
\end{abstract}

DOI: 10.1103/PhysRevB.90.205307

PACS number(s): 78.47.D-, 78.67.De

\section{INTRODUCTION}

There has been a great deal of interest in the formation processes of resident electron-spin polarization (RESP) confined in nanostructures for potential applications in future semiconductor electro-optic devices and quantum information processing [1-5]. These future applications could benefit from a long spin coherence of RESP because RESP can exist after the recombination of an associated optically excited state such as negatively charged excitons (trions) and the coherence is not limited by the fast recombination process. A lot of research effort has been put forth to understand better the interplay among resident electrons, neutral excitons, trions, and donor bound excitons [6-11], and with their environments such as tunable lasers [12] and lattice nuclei [13]. Based on those efforts, the correct perspective of the evolution of the optically excited states allows for better controlling the electron spins $[11,14]$. However, it is still difficult to capture the exact snapshots of the ultrafast transforming process of the excited states during the RESP generation. In addition, the physical interpretation of initial phases in the RESP transient signals has not been fully established.

In this paper, we study the dynamical process of RESP of a CdTe single quantum well (SQW) in recognizing the importance of the initial phase shift (IPS) with a time-resolved Kerr rotation (TRKR) technique. The careful analysis of the TRKR signals as compared to the theoretical fittings around the time origin $(t=0)$ reveals an appealing phenomenon: the negative IPS by which one looks "backward" in the time domain toward zero delay as shown later (Fig. 3). Since the IPS gives the important clues about the generation processes of RESP, the experimental and theoretical studies of IPS play a crucial role in the study of RESP.

Although the positive and zero IPSs have been reported in CdTe QWs [15,16] so far, we observed the negative IPS in a CdTe SQW. In addition, we found that the magnitude of IPS can be controlled by the external parameters such as the excitation power and the strength of the external magnetic field.

\footnotetext{
*adachi-s@eng.hokudai.ac.jp
}

By using the model calculation which involves the interplay between the trion and RESP, we discuss the possibility of the negative IPS and dynamical process of RESP in detail.

\section{EXPERIMENTAL DETAIL}

The sample used in this study was a naturally $n$ doped $\mathrm{CdTe} / \mathrm{Cd}_{0.85} \mathrm{Mg}_{0.15} \mathrm{Te} \mathrm{SQW}$ with the well width of $100 \AA$ grown by molecular-beam epitaxy on a (100)-oriented GaAs substrate. The natural doping due to impurities in barriers causes a background resident electron ensemble $\left(n_{e} \sim 10^{10} \mathrm{~cm}^{-2}\right)$, which can interact with optically injected electron heavy-hole (e-hh) pairs to form negative trions in the ground state. Figure 1 shows photoluminescence (PL) spectra at $10 \mathrm{~K}$. The PL spectra can be fitted by the sum of the two Lorentzian functions and both the negative trions $(T)$ (the low-energy peak at $1.6110 \mathrm{eV}$ ) and the neutral hh excitons $\left(X^{0}\right)$ (the high-energy peak at $1.6133 \mathrm{eV}$ ) are clearly separated by the trion binding energy of $2.3 \mathrm{meV}$, which is beneficial to excite trions selectively. This trion is the lowest-energy optical transition in the system.

The dynamical evolution of the created RESP and the interplay with trions were measured by the TRKR technique. Both the pump and probe pulses were produced by a modelocked Ti:sapphire laser with the pulse width of $\sim 2$ ps and the repetition rate of $76 \mathrm{MHz}$. Since the transform from excitons to trions makes the evaluation of IPS complicated, the laser wavelength was tuned to the negative trion resonance at $769.6 \mathrm{~nm}$ as seen in the PL spectra. The spectral width of the pump and probe pulses $(<1 \mathrm{meV})$ was narrower compared to the trion spectrum. Under this excitation condition, the excitation of the exciton was suppressed strongly. A circularly polarized $\left(\sigma^{+}\right.$or $\sigma^{-}$) pump pulse was incident along the QW growth direction $(z)$ and magnetic fields up to $\sim 720 \mathrm{mT}$ were applied in the $x$ direction (Voigt geometry). In this geometry, the spins were prepared initially orthogonal to an applied field, and started to precess with Larmor frequency $\omega_{\mathrm{L}}$ in the $y z$ plane immediately after optical excitation. The pump pulse was modulated from $\sigma^{+}$to $\sigma^{-}$at $50 \mathrm{kHz}$ to reduce the laser noise. Similar to a standard TRKR setup [17,18], a linearly 


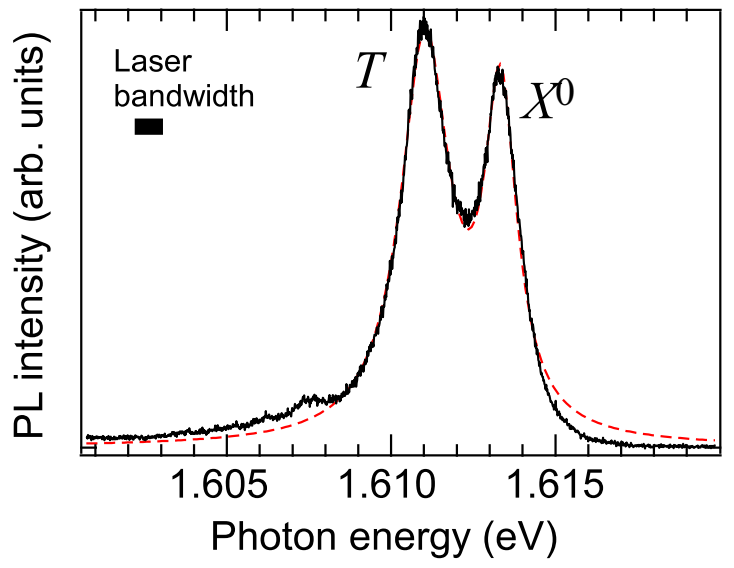

FIG. 1. (Color online) PL spectra of the studied $\mathrm{CdTe} /(\mathrm{Cd}$, $\mathrm{Mg}$ )Te SQW at $10 \mathrm{~K}$ with the excitation laser energy of $1.65 \mathrm{eV}$. The negative trion $(T)$ and neutral exciton $\left(X^{0}\right)$ are clearly separated by a trion binding energy $(2.3 \mathrm{meV})$. The dashed line indicates a fitting by Lorentzian shaped spectra. The excitation bandwidth is also indicated by a thick bar.

polarized probe pulse with a controlled delay time monitors the electron-spin polarization in the spot area excited by a pump pulse. A Kerr rotation of the linear polarization was measured sensitively using a polarization bridge and double lock-in detection technique. The QW sample was mounted in an optical cryostat and the temperature was kept at $\sim 10 \mathrm{~K}$ through all the measurements.

Based on a reported diffusion constant of the negative trions $D \sim 10 \mathrm{~cm}^{2} / \mathrm{s}$ at $10 \mathrm{~K}$ studied via transient grating experiments [19], we assumed reasonably that the excited carriers could not escape out of a probed spot $(\sim 100-\mu \mathrm{m}$ diameter) during the observed time range $(\sim 3 \mathrm{~ns})$, and therefore the electron diffusion was not taken into account in this study.

\section{RESP DYNAMICS AND MODELING}

\section{A. General spin dynamics and initial phase shift of RESP}

A schematic of the general RESP dynamics on the basis of prior studies $[16,20]$ is depicted in Fig. 2. The figure is depicted under the condition that the trion recombination time is smaller than the precession period: $\tau_{\mathrm{r}}^{T}<2 \pi / \omega_{\mathrm{L}}$. In the presence of background resident electrons, before optical excitation (stage I), there is no RESP and individual electron spins rotate around the applied magnetic field $B_{x}$. At the time origin ( $t=0$, stage II), optical excitation ( $\sigma^{-}$excitation in the figure) at the trion resonance can generate an e-hh pair $(\uparrow \Downarrow)$ selectively, which captures one electron with opposite spin $(\downarrow)$ from the resident electron ensemble and forms a negative trion $(\downarrow \uparrow \Downarrow)$. At this moment, the resident electron ensemble loses the spin balance and a nonzero net RESP comes out along the light propagation direction (stage III).

The return of the electron after trion recombination can change RESP if the conditions are right. If the hole spin relaxation time $\tau_{\mathrm{s}}^{T}$ of the negative trion is longer than $\tau_{\mathrm{r}}^{T}$, an electron with the same $\operatorname{spin}(\downarrow)$ as that captured at $t=0$ returns to the resident electron ensemble (stage IV). In the case

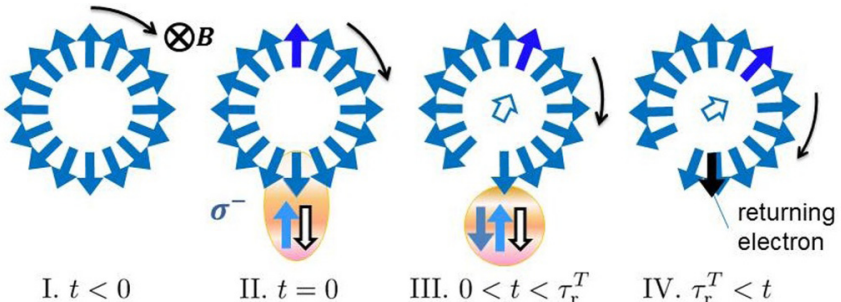

FIG. 2. (Color online) Schematic of the RESP dynamics, where interplay between resident electrons and the optically excited negative trion are illustrated. I: The resident electron spins with the nonpolarized initial state (arrows spread in a circle) are precessing around the magnetic field $B_{x}$. Note that the direction of the individual arrows in the figure represents only the probability of spin up or spin down when it is observed. II: The e-hh $(+1 / 2,-3 / 2)$ pair generated by a $\sigma^{-}$excitation captures an electron with the proper spin direction (in this case $-1 / 2$ ) from the resident electron ensemble automatically according to the Pauli exclusion principle to form a negative trion. At the moment, the net polarization in the resident electron ensemble is created and the IPS of the RESP is zero. III: Since a trion consists of a spin-singlet electron pair and a hole (in-plane hole $g=0$ ), the trion does not precess in the magnetic field. The hole spin may flip during the trion recombination time $\left(\tau_{\mathrm{r}}^{T}\right)$. IV: The electron spin left behind by the trion recombination returns back to the ensemble of resident electrons, and it changes the net RESP and the IPS.

of $\tau_{\mathrm{s}}^{T}<\tau_{\mathrm{r}}^{T}$, an electron with the opposite spin may return. Therefore, depending on $\tau_{\mathrm{s}}^{T}, \tau_{\mathrm{r}}^{T}$, and the precession period $2 \pi / \omega_{\mathrm{L}}$, the amplitude and phase of the TRKR signal originated from RESP may change intricately. The microscopic picture of the above RESP generation is given by Kennedy et al. [21] using a coherent superposition of electrons and trion states excited by circularly polarized excitation under a transverse magnetic field.

A typical one-color TRKR signal for the studied CdTe SQW is shown in Fig. 3. The signal was taken by a $\sigma^{-}$-pulsed excitation with low power $\left(P_{\text {pump }}=1 \mathrm{~mW}\right)$ at the trion resonance under $B_{x}=146 \mathrm{mT}$ and $10 \mathrm{~K}$. The TRKR signal

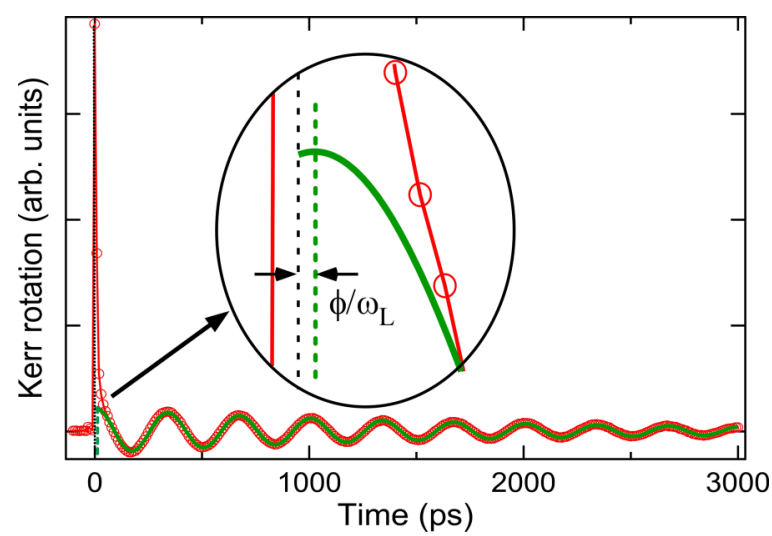

FIG. 3. (Color online) A typical TRKR signal (146 mT and 10 K) and the fitting of the RESP component (solid line). The RESP shows a long coherence time of $\tau_{s}^{e} \sim 1800$ ps. Inset: The negative IPS $\phi$ of RESP is shown by a backward temporal shift $\phi / \omega_{\mathrm{L}}=-8.1 \mathrm{ps}$. The signal was taken at the low intensity excitation $\left(P_{\text {pump }}=1 \mathrm{~mW}\right)$. 
can be fitted by the following function:

$$
S(t)=A^{T} \exp \left(-\frac{t}{\tau^{T}}\right)+A^{e} \exp \left(-\frac{t}{\tau_{s}^{e}}\right) \cos \left(\omega_{L} t+\phi\right),
$$

where the coefficients $A^{T}$ and $A^{e}$ are the amplitudes of the TRKR signals originated from trions and RESP, $\tau^{T}$ and $\tau_{s}^{e}$ are the corresponding spin lifetimes, and $\phi$ is the initial phase of RESP precession. Since a negative trion consists of spin-paired electrons (singlet state) and a hole, the total $g$ factor in the $x$ direction is zero in $\mathrm{QW}$, and therefore negative trions show a simple decay due to the short spin lifetime $\tau^{T}$ without the spin precession by a magnetic field as indicated by the first term of Eq. (1). Note that the in-plane hh $g$ factor is nearly zero in a QW as long as light hole (lh)-hh mixing is weak [22].

Spin lifetime of trions is defined as a combination of the recombination time and the transverse hole spin-relaxation time $1 / \tau^{T}=1 / \tau_{\mathrm{r}}^{T}+1 / \tau_{\mathrm{s}}^{T}$. On the other hand, the spin lifetime of the RESP is determined only by the transverse spinrelaxation time $\tau_{\mathrm{s}}^{e}$. A long coherence time is expected for RESP compared with the trion, and in fact the RESP component represented by the second term of Eq. (1) shows a long $\tau_{s}^{e} \sim 1800 \mathrm{ps}$ in Fig. 3. Note that the experimentally measured $\tau_{\mathrm{s}}^{e}$ in the TRKR technique is the transverse spin-relaxation time for the macroscopic number of electron spins but not the intrinsic spin coherence time of the individual electron. This macroscopic spin relaxation includes the dephasing effect due to some inhomogeneities such as electron $g$-factor distribution and local magnetic field. The RESP component indicates the precession with $\omega_{\mathrm{L}}$ given by $g_{\perp}^{e} \mu_{\mathrm{B}} B_{x} / \hbar$, where $g_{\perp}^{e}$ is an in-plane electron $g$ factor, $\mu_{\mathrm{B}}$ is the Bohr magneton $(\sim 58 \mu \mathrm{eV} / \mathrm{T})$, and $\hbar$ is the reduced Plank constant. In the studied CdTe SQW, $\left|g_{\perp}^{e}\right|=1.469 \pm 0.003$ was obtained from the magnetic field dependence of $\omega_{L}$.

The TRKR signals given by Eq. (1) become generally complicated particularly around $t=0$, but it is noted that the first term disappears rapidly due to the shorter spin lifetime compared to that of RESP. The recombination time of trions obtained by the time-resolved PL measurements using a streak camera was $\tau_{\mathrm{r}}^{T}=85 \pm 1 \mathrm{ps}$, and $\tau_{\mathrm{s}}^{T}$ is much shorter than $\tau_{\mathrm{r}}^{T}$ as the signal indicates. The measured $\tau_{\mathrm{r}}^{T}$ is similar to the values in the previous reports [10]. Thus, only the RESP component with a long coherence survives after a few 100 ps. Since the spin decay in the probe volume caused by the diffusion process can be neglected, the envelope of the TRKR signal in the long delay-time region reflects the dephasing of RESP directly.

In Fig. 3, the fitting for the range of 500-3000 ps in a TRKR signal by the second term of Eq. (1) is indicated by a solid line. As indicated in the inset, the interpolating back to $t=0$ can be seen as the time shift $\phi / \omega_{L}(=-8.1 \mathrm{ps})$ of RESP generation. The time shift can be converted to IPS $=-0.15 \mathrm{rad}$. Although the time shift and the IPS are small in the signal, the appearance of the negative IPS was always observed in our sample at various conditions. Then, we emphasize that the observed IPS is intrinsic and is disentangled unambiguously from the measurement-induced unphysical time delay in a pump-probe setting. This negative IPS is a main subject of this study, and the dependences on excitation power and magnetic field strength are presented in Sec. IV.

\section{B. Modeling of RESP generation}

To gain significant insight into the IPS mechanism and to analyze our experimental results quantitatively, we put forth a theoretical model for the spin dynamics of RESP. The model contains three processes ( $\mathrm{P} 1, \mathrm{P} 2$, and $\mathrm{P} 3)$ to generate the RESP, where the excitation at the trion resonance is assumed and the excitation of the neutral excitons is excluded for simplicity.

In the P1 process, the e-hh pairs $(\uparrow \Downarrow)$ are created by absorption of the $\sigma^{-}$photons at $t=0$ and capture $-1 / 2$ $(\downarrow)$ electrons from the resident electron ensemble to form $-3 / 2$ trions $\left(T_{\Downarrow} ; \downarrow \uparrow \Downarrow\right)$ with a concentration $N_{0}$. This electron capturing is equivalent to leaving behind electrons of $+1 / 2(\uparrow)$ with the same concentration in the resident electron ensemble, and therefore the initial RESP can form by this P1 process. Since the pulse width (2 ps) is much shorter than the spin precession period $2 \pi / \omega_{\mathrm{L}}$, the spin rotation during the pulse duration can be neglected. Thus, the P1 process does not induce a finite nonzero IPS due to the instantaneous excitation of RESP.

The generated RESP $S^{e}(t)\left\{\equiv\left[S^{e_{\uparrow}}(t)-S^{e_{\downarrow}}(t)\right] / 2\right\}$ follows the Bloch equation including the relaxation terms:

$$
\frac{d \boldsymbol{S}^{e}}{d t}=\omega_{\mathrm{L}} \times \boldsymbol{S}^{e}-\frac{\boldsymbol{S}^{e}}{\tau_{\mathrm{s}}^{e}}+\frac{\boldsymbol{S}^{T}}{\tau_{\mathrm{r}}^{T}},
$$

where $\boldsymbol{S}^{T}(t)\left\{\equiv\left[\boldsymbol{S}^{T_{\Uparrow}}(t)-\boldsymbol{S}^{T_{\Downarrow}}(t)\right] / 2\right\}$ is the trion spin polarization, and the initial conditions are $S^{e}(0)=\left(0,0, N_{0} / 2\right)$, $\boldsymbol{S}^{T}(0)=\left(0,0,-N_{0} / 2\right)$, and $\boldsymbol{\omega}_{\mathrm{L}}=\left(\omega_{\mathrm{L}}, 0,0\right)$.

The other two RESP generation processes P2 and P3 occur via the recombination of $T_{\Downarrow}$ and $T_{\Uparrow}$, respectively, which are represented as the third term of Eq. (2). The recombination of $T_{\Downarrow}$ returns $-1 / 2(\downarrow)$ electrons back to the resident electron ensemble (P2 process), while $T_{\Uparrow}$ returns $+1 / 2(\uparrow)$ electrons by the recombination ( $\mathrm{P} 3$ process). Since $T_{\Downarrow}$ is generated initially by optical excitation, the hole spin flip in $T_{\Downarrow}$ is required in the P3 process. Note that the condition $2 \pi / \omega_{\mathrm{L}}>\tau_{\mathrm{r}(\mathrm{s})}^{T}$ is assumed below according to our experiments. Under the condition $2 \pi / \omega_{\mathrm{L}}<\tau_{\mathrm{r}(\mathrm{s})}^{T}$, many events of the hole spin flip and recombination occur in one spin rotation period, and P2 and P3 have no contribution to RESP and therefore to IPS.

The trion dynamics can be represented simply as $d \boldsymbol{S}^{T} / d t=$ $-\boldsymbol{S}^{T} / \tau^{T}$. Recalling $\tau^{T}$ is defined as $1 / \tau^{T}=1 / \tau_{\mathrm{r}}^{T}+1 / \tau_{\mathrm{s}}^{T}$, in the case of $\tau_{\mathrm{s}}^{T}>\tau_{\mathrm{r}}^{T}$, P2 is a major process compared to P3. This process makes the IPS of RESP positive due to the return of $-1 / 2(\downarrow)$ electrons. On the other hand, the case $\tau_{\mathrm{s}}^{T} \ll \tau_{\mathrm{r}}^{T}$ induces the equivalent return of $+1 / 2$ and $-1 / 2$ electrons to the resident electron ensemble, and therefore IPS of RESP does not change from zero.

To explain the observed negative IPS, it is necessary that $\mathrm{P} 3$ becomes a major one in the electron returning process. To simplify the modeling, via P2 and P3 processes, we assume that spin-polarized trion populations of $T_{\Downarrow}$ and $T_{\Uparrow}$ evolve as $S^{T_{\Downarrow}}=-N_{0} \exp \left(-t / \tau_{\mathrm{r}}^{T}\right) \exp \left(-t / \tau_{\mathrm{s}}^{T}\right)$ and $S^{T_{\Uparrow}}=N_{0} \exp \left(-t / \tau_{\mathrm{r}}^{T}\right)+S^{T_{\Downarrow}}$. This hole spin-relaxation process is asymmetric between $T_{\Downarrow} \rightarrow T_{\Uparrow}$ and $T_{\Uparrow} \rightarrow T_{\Downarrow}$, and the mechanism was discussed in the spin-flip Raman scattering measurements by Debus et al. [23]. The assumption should be tested by the comparison between the experimental and calculated results. 
Assuming the above initial conditions, the following are obtained to solve Eq. (2) including the above asymmetric hole spin flip:

$$
\begin{aligned}
S_{P 1}^{e}(t) & =\frac{N_{0}}{2} \cos \left(\omega_{L} t\right) \exp \left(-\frac{t}{\tau_{s}^{e}}\right), \\
S_{P 2+P 3}^{e}(t) & =\int_{0}^{t} \frac{S^{T}\left(t^{\prime}\right)}{\tau_{r}^{T}} \cos \left\{\omega_{L}\left(t-t^{\prime}\right)\right\} \exp \left(-\frac{t-t^{\prime}}{\tau_{s}^{e}}\right) d t^{\prime} .
\end{aligned}
$$

Then, the RESP $S^{e}(t)\left[=S_{P 1}^{e}(t)+S_{P 2+P 3}^{e}(t)\right]$ is

$$
\begin{aligned}
S^{e}(t)= & {\left[A \cos \left(\omega_{\mathrm{L}} t\right)+B \sin \left(\omega_{\mathrm{L}} t\right)\right] \exp \left(-\frac{t}{\tau_{\mathrm{s}}^{e}}\right) } \\
& +C \exp \left(-\frac{t}{\tau_{\mathrm{r}}^{T}}\right)+D \exp \left\{-t\left(\frac{1}{\tau_{\mathrm{s}}^{T}}+\frac{1}{\tau_{\mathrm{r}}^{T}}\right)\right\}, \\
A=\frac{N_{0}}{2} & {\left[1+\frac{1}{1+\left(\omega_{\mathrm{L}} \tau_{\mathrm{r}}^{T}\right)^{2}}-\frac{2 \tau_{\mathrm{s}}^{T}\left(\tau_{\mathrm{r}}^{T}+\tau_{\mathrm{s}}^{T}\right)}{\left(\tau_{\mathrm{r}}^{T}+\tau_{\mathrm{s}}^{T}\right)^{2}+\left(\omega_{\mathrm{L}} \tau_{\mathrm{s}}^{T} \tau_{\mathrm{r}}^{T}\right)^{2}}\right], } \\
B=\frac{N_{0}}{2} & {\left[\frac{\omega_{\mathrm{L}} \tau_{\mathrm{r}}^{T}}{1+\left(\omega_{\mathrm{L}} \tau_{\mathrm{r}}^{T}\right)^{2}}-\frac{2 \omega_{\mathrm{L}} \tau_{\mathrm{r}}^{T}\left(\tau_{\mathrm{s}}^{T}\right)^{2}}{\left(\tau_{\mathrm{r}}^{T}+\tau_{\mathrm{s}}^{T}\right)^{2}+\left(\omega_{\mathrm{L}} \tau_{\mathrm{s}}^{T} \tau_{\mathrm{r}}^{T}\right)^{2}}\right], } \\
C=- & \frac{N_{0} / 2}{1+\left(\omega_{\mathrm{L}} \tau_{\mathrm{r}}^{T}\right)^{2}}, \quad D=\frac{N_{0} \tau_{s}^{T}\left(\tau_{r}^{T}+\tau_{\mathrm{s}}^{T}\right)}{\left(\tau_{\mathrm{r}}^{T}+\tau_{\mathrm{s}}^{T}\right)^{2}+\left(\omega_{\mathrm{L}} \tau_{\mathrm{s}}^{T} \tau_{\mathrm{r}}^{T}\right)^{2}} .
\end{aligned}
$$

In the derivation of the coefficients $A, B, C$, and $D$ in Eqs. (4)(6), we ignored the higher-order correction terms of $\left(1 / \tau_{\mathrm{s}}^{e}\right)^{\mathrm{n} \geqslant 1}$ that play a negligible role in the results since the electron-spinrelaxation time is much longer than the trion's time constants $\left(\tau_{\mathrm{s}}^{e} \gg \tau_{\mathrm{r}}^{T}>\tau_{\mathrm{s}}^{T}\right)$. Finally, the IPS $\phi$ and the amplitude $P$ are obtained as follows:

$$
\begin{gathered}
\phi=\arctan (-B / A), \\
P=\sqrt{A^{2}+B^{2}} .
\end{gathered}
$$

\section{RESULTS AND ANALYSIS}

For a deeper insight into the interplay between the dynamical evolution of trions and the resultant IPS of RESP, the TRKR signals under $B_{x}=146 \mathrm{mT}$ (precession period $2 \pi / \omega_{\mathrm{L}}=333 \mathrm{ps}$ ) were measured as a function of the pump power over the range of 1-10 $\mathrm{mW}$ as shown in Fig. 4(a). The fitting for the RESP component in $t=500-3000 \mathrm{ps}$ indicates that $\tau_{\mathrm{s}}^{e}$ decreases from 1800 to $800 \mathrm{ps}$ with increasing the pump power. A possible reason for the reduction of $\tau_{\mathrm{s}}^{e}$ is the increase of phonon scattering due to the heating of resident electrons by optical excitation.

Figure 4(b) summarizes the IPS values of these TRKR signals in Fig. 4(a). For all the pump powers, the negative IPS was observed and the absolute value mainly increases and then saturates with the increasing pump power. The maximum negative IPS of $\sim-0.35 \mathrm{rad}$ was obtained at the pump power of $P_{\text {pump }} \sim 8 \mathrm{~mW}$. By using Eqs. (4), (5), and (7), IPS is calculated as functions of $\tau_{\mathrm{s}}^{T}$ and $\tau_{\mathrm{r}}^{T}$ under the experimentally used $B_{x}=146 \mathrm{mT}$ [Fig. 4(c)]. It is found
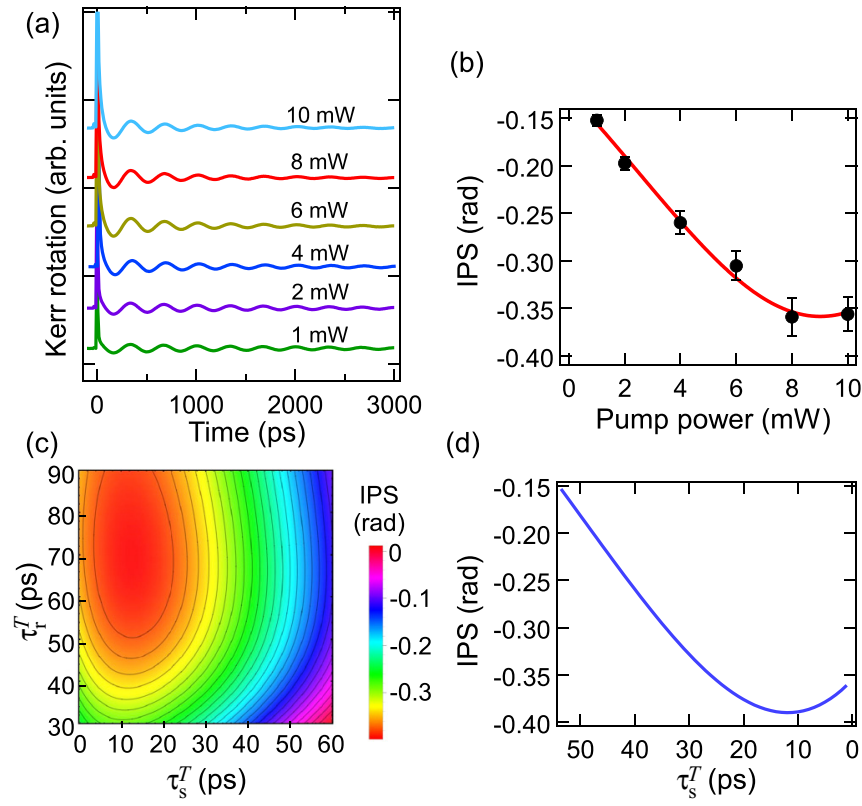

FIG. 4. (Color online) (a) TRKR signals as a function of pump power $(146 \mathrm{mT}, 10 \mathrm{~K})$. (b) Experimental IPS (symbols) of RESP with varying pump power (the solid line is a guide for the eye). (c) The calculated IPS contour plot as functions of the recombination time $\tau_{\mathrm{r}}^{T}$ and the hole spin-relaxation time $\tau_{\mathrm{s}}^{T}$. (d) The theoretical IPS as a function of $\tau_{\mathrm{s}}^{T}$, which is a slice of (c) at $\tau_{\mathrm{r}}^{T}=85 \mathrm{ps}$.

that the negative IPS is obtained in the region of $\tau_{\mathrm{s}}^{T}<\tau_{\mathrm{r}}^{T}$ and IPS comes to zero when $\tau_{\mathrm{s}}^{T}$ approaches to $\tau_{\mathrm{r}}^{T}$. Figure 4(d) is a slice of the two-dimensional plot at $\tau_{\mathrm{r}}^{T}=85 \mathrm{ps}$, which was practically obtained from the independent time-resolved PL measurements. If the relation between the pump power (trion density) and the hole spin-relaxation time is known, the data in Figs. 4(b) and 4(d) can compare directly, but the saturation point on the IPS is a better choice in the evaluation of $\tau_{\mathrm{s}}^{T}$ at the present stage. Obviously, the maximum negative IPS is $\sim-0.39 \mathrm{rad}$ at $\tau_{\mathrm{s}}^{T} \sim 15 \mathrm{ps}$ in the calculated result at $B_{x}=$ $146 \mathrm{mT}$ and agrees well with the experimentally obtained result of $-0.35 \mathrm{rad}$.

In order to verify further that the IPS is negative in this QW sample, the following experiment was performed. Since the change of the IPS definitely originates from the subsequently generated RESP component, for example, due to trion recombination, the use of the controllable extra RESP component is beneficial, and more precise measurement of the intrinsic IPS can be expected. In this measurement, the controllable extra RESP component was produced by an additional circularly polarized pump pulse (called a control pulse, here). Figure 5 shows the TRKR signals excited by the $\sigma^{-}$pump with (a) $\sigma^{-}$and (b) $\sigma^{+}$control pulses that were denoted by $\sigma^{-} / \sigma^{-}$and $\sigma^{-} / \sigma^{+}$, respectively, under $B_{x}=136 \mathrm{mT}$ and $10 \mathrm{~K}$. The control pulse was retarded by $\pi / 2$ (around $90 \mathrm{ps)}$ ) and was varied from 0.5 to $10 \mathrm{~mW}$ for both cases. For the constant RESP generated by the first pump pulse, the control pulse produces the extra RESP with the relative phase $\pi / 2$ for $\sigma^{-}$and $3 \pi / 2$ for $\sigma^{+}$. The relative phase shift $\Phi$ of the combined total RESP can be varied by changing the control power as indicated in the insets of Figs. 5(a) 


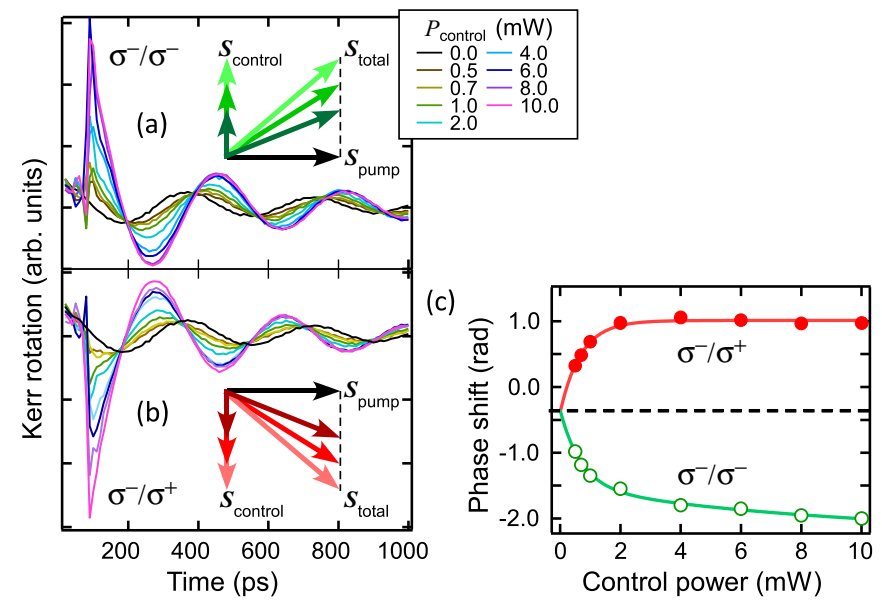

FIG. 5. (Color online) TRKR signals obtained by both the $\sigma^{-}$ pump of $10 \mathrm{~mW}$ and (a) $\sigma^{-}$or (b) $\sigma^{+}$control with different excitation power and the delay by $\pi / 2(136 \mathrm{mT}, 10 \mathrm{~K})$. (c) The corresponding phase shifts from the fitting of the signals (a) (green) and (b) (red). Both the phase shifts converge to $-0.38 \mathrm{rad}$ in the limit of zero control power.

and 5(b). The $|\Phi|$ is expected to shift down with decreasing the extra RESP component and to converge to the intrinsic IPS produced by the first pump pulse in the limit of the zero control power.

The $\Phi$ of the RESP precessions are plotted in Fig. 5(c). The $\Phi$ was obtained from the fitting over the range of 500-3000 ps. As shown in Fig. 5(c), each absolute phase shift $\left|\Phi_{-}\right|$for the $\sigma^{-} / \sigma^{-}$signal is much larger than $\left|\Phi_{+}\right|$of the $\sigma^{-} / \sigma^{+}$curve under the same power of the control pulse. The $\left|\Phi_{-}\right|$and $\left|\Phi_{+}\right|$change and converge to the same nonzero phase shift $(-0.38 \mathrm{rad})$ at $P_{\text {control }}=0 \mu \mathrm{W}$ consistently, corresponding to the phase shift of the original signal excited by the single pump pulse and also similar to the IPS ( $-0.35 \mathrm{rad})$ in Fig. 4(b). In addition, as seen from the curves of $\left|\Phi_{-}\right|$and $\left|\Phi_{+}\right|$, they are not strictly mirror symmetric about the horizontal dashed line of $\Phi=-0.38$. This is due to the existence of the intrinsic IPS caused by the first pump pulse excitation as well as the varying intrinsic IPS induced by the control pulse.

Finally, we show a set of TRKR curves detected under the various magnetic fields $B_{x}$ up to $\sim 720 \mathrm{mT}$ and $P_{\text {pump }}=8 \mathrm{~mW}$ in Fig. 6(a). The used $B_{x}$ corresponds to $\omega_{\mathrm{L}}=0.45-9.24 \times$ $10^{-2} \mathrm{rad} / \mathrm{ps}$ (period 1390-68 ps). The RESP components were fitted over the time range of $t=500-3000$ ps. Obviously, the RESP decays faster with increasing $B_{x}$ ( $\tau_{\mathrm{s}}^{e}$ changes from 1185 to $500 \mathrm{ps}$ ). This reduction of $\tau_{\mathrm{s}}^{e}$ is attributed to the averaging effect of a macroscopic number of spins having the inhomogeneity of the $g$ factor due to the fluctuation of well width and alloy composition of the barrier material. A similar dephasing effect was reported in Refs. [11,20].

From these TRKR signals, the dependence of the IPS on Larmor frequency is plotted by the solid circles in Fig. 6(b). The absolute value of the negative IPS first increases only in the small Larmor frequency range (i.e., $\omega_{\mathrm{L}} \leqslant 2 \times 10^{-2} \mathrm{rad} / \mathrm{ps}$, $B_{x} \leqslant 200 \mathrm{mT}$ ) and then decreases with increasing $\omega_{\mathrm{L}}$. What is more, their RESP amplitude decreases at first and then goes up gradually with increasing $\omega_{\mathrm{L}}$ as indicated by Fig. 6(c).
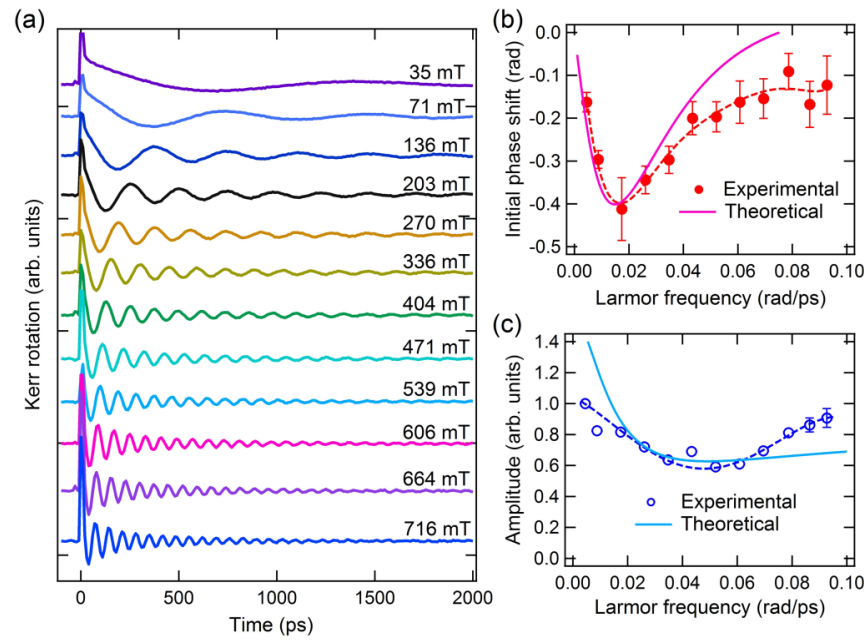

FIG. 6. (Color online) (a) The dependence of the TRKR signals on $B_{x}$ in the range of $35-716 \mathrm{mT}$ at $P_{\text {pump }}=8 \mathrm{~mW}$. (b) The corresponding IPS (solid circles) and the simulated IPS denoted by a solid line. (c) The corresponding relative amplitude (open circles) and the calculated amplitude denoted by a solid line. The calculated IPS and amplitude are plotted as a function of Larmor frequency under $\tau_{\mathrm{s}}^{T}=15 \mathrm{ps}$ and $\tau_{\mathrm{r}}^{T}=85 \mathrm{ps}$. The dashed lines in (b) and (c) are guides for the eye.

The IPS and amplitude of Kerr rotation in dependence on $\omega_{\mathrm{L}}$ from the model calculation were also shown by a solid line in the respective figures. For the model calculation, we took $\tau_{\mathrm{r}}^{T}=85 \mathrm{ps}$ and $\tau_{\mathrm{s}}^{T}=15 \mathrm{ps}$ mentioned above. Theoretical IPS reproduces well the experimental results in the small $\omega_{\mathrm{L}}$ region and deviates a little to smaller values in the large $\omega_{\mathrm{L}}$ region. In addition, the variation trend of theoretical amplitude displays a similarity with that of experimental results. Although the deviation in the large $\omega_{\mathrm{L}}$ region in both figures cannot be explained in the framework of our simple model at present, the overall trend of the $\omega_{\mathrm{L}}$ dependences of IPS and amplitude of RESP was found to be applicable to explain the experimental results.

All the above experimental results indicate that there is the obvious negative IPS at various conditions. The negative IPS can be brought up as far as there is an asymmetric fast hole spin flip from $T^{\Downarrow}$ to $T^{\Uparrow}$ under $\sigma^{-}$polarized excitation, and vice versa for $\sigma^{+}$excitation, especially shorter than recombination time. Comparison of the experimental data with theory shows that the assumption is in good agreement with the experimental findings although we could not specify the physical origin at the present stage. The calculation model presented in this work is just a simple model capturing the essential physics of trion and electron-spin-polarization evolutions.

It is well known that CdTe is one of the attractive compounds in terms of smaller effects of hyperfine interaction and quadrupolar interaction induced by strain due to small abundance of the isotopes with one-half nuclear spin [24,25], compared to III-V semiconductors such as GaAs and InAs. Thus, the hole and electron-spin dynamics are expected to be affected far less by the hyperfine interaction with nuclear spins even in higher confined structures (e.g., quantum dots). However, to build the theory up into a specifically and fully microscopic model, a detailed understanding of confining 
potential, kinetic energy for holes, degree of hh-lh mixing, and anisotropic exchange interaction for II-VI QWs might be required.

\section{CONCLUSIONS}

To summarize, we have successfully demonstrated a transient IPS process via systematically investigating the precessional traces of RESP involving the trion spin dynamics in a CdTe SQW in a rather low magnetic field region by using a TRKR technique. All the above experimental results indicate that there was remarkable negative IPS for all TRKR signals, which was identified as a result of the intrinsic spin-polarization dynamics, especially confirmed by more proof of the double pump excitation experiments, as discussed above. The results of the model calculation agree well with the experimental factors for the overall trends of IPSs. Most interestingly, through the interpretation of the nonzero IPSs in the RESP precessions, the trion spin dynamical process and the RESP formation can be determined in the calculation as well as in the experiments, where the briskly asymmetric hole spin flip accounts for the negative initial phase. To get a clear physical picture of all the dynamics, further research about the trion spin dynamics is required. However, we believe that the results we presented here will be valuable and can contribute to better manipulation and understanding of the RESP and charged exciton dynamics.

\section{ACKNOWLEDGMENTS}

S.A. is grateful to Wei-Ting Hsu and Wen-Hao Chang of National Chiao Tung University for fruitful discussions. This work was supported in part by JSPS KAKENHI (Grants No. 22310063 and No. 25247047). Also, the China Scholarship Council is acknowledged for the studentship financial support at Hokkaido University.
[1] D. D. Awschalom, N. Samarth, and D. Loss, Semiconductor Spintronics and Quantum Computation (Springer-Verlag, Berlin, 2002).

[2] Spin Physics in Semiconductors, edited by M. I. Dyakonov, Springer Series in Solid-State Sciences Vol. 157 (SpringerVerlag, Berlin, 2008), Chaps. 2, 3, and 6.

[3] M. M. Glazov, Phys. Solid State 54, 1 (2012).

[4] S. Crankshaw, F. G. Sedgwick, M. Moewe, C. Chang-Hasnain, H. L. Wang, and S. L. Chuang, Phys. Rev. Lett. 102, 206604 (2009).

[5] W. H. Lau and M. E. Flatte, J. Appl. Phys. 91, 8682 (2002).

[6] R. T. Harley, M. A. Brand, A. Malinowski, O. Z. Karimov, P. A. Marsden, A. J. Shields, D. Sanvitto, D. A. Ritchie, and M. Y. Simmons, Physica E 17, 324 (2003).

[7] Z. Chen, R. Bratschitsch, S. G. Carter, S. T. Cundiff, D. R. Yakovlev, G. Karczewski, T. Wojtowicz, and J. Kossut, Phys. Rev. B 75, 115320 (2007).

[8] W. Ossau, D. R. Yakovlev, C. Y. Hu, V. P. Kochereshko, G. V. Astakhov, R. A. Suris, P. C. M. Christianen, and J. C. Maan, Phys. Solid State 41, 751 (1999).

[9] E. Vanelle, M. Paillard, X. Marie, T. Amand, P. Gilliot, D. Brinkmann, R. Levy, J. Cibert, and S. Tatarenko, Phys. Rev. B 62, 2696 (2000).

[10] J. Tribollet, F. Bernardot, M. Menant, G. Karczewski, C. Testelin, and M. Chamarro, Phys. Rev. B 68, 235316 (2003).

[11] J. Tribollet, E. Aubry, G. Karczewski, B. Sermage, F. Bernardot, C. Testelin, and M. Chamarro, Phys. Rev. B 75, 205304 (2007).

[12] P. Palinginis and H. Wang, J. Magn. Magn. Mater. 272-276, 1919 (2004).

[13] G. Salis, D. D. Awschalom, Y. Ohno, and H. Ohno, Phys. Rev. B 64, 195304 (2001).
[14] C. Phelps, T. Sweeney, R. T. Cox, and H. Wang, Phys. Rev. Lett. 102, 237402 (2009).

[15] C. Camilleri, F. Teppe, D. Scalbert, Y. G. Semenov, M. Nawrocki, M. Dyakonov, J. Cibert, S. Tatarenko, and T. Wojtowicz, Phys. Rev. B 64, 085331 (2001).

[16] E. A. Zhukov, D. R. Yakovlev, M. Bayer, M. M. Glazov, E. L. Ivchenko, G. Karczewski, T. Wojtowicz, and J. Kossut, Phys. Rev. B 76, 205310 (2007).

[17] J. J. Baumberg, S. A. Crooker, D. D. Awschalom, N. Samarth, H. Luo, and J. K. Furdyna, Phys. Rev. B 50, 7689 (1994).

[18] S. A. Crooker, D. D. Awschalom, J. J. Baumberg, F. Flack, and N. Samarth, Phys. Rev. B 56, 7574 (1997).

[19] M. T. Portella-Oberli, V. Ciulin, S. Haacke, J.-D. Ganière, P. Kossacki, M. Kutrowski, T. Wojtowicz, and B. Deveaud, Phys. Rev. B 66, 155305 (2002).

[20] E. A. Zhukov, D. R. Yakovlev, M. Gerbracht, G. V. Mikhailov, G. Karczewski, T. Wojtowicz, J. Kossut, and M. Bayer, Phys. Rev. B 79, 155318 (2009).

[21] T. A. Kennedy, A. Shabaev, M. Scheibner, A. L. Efros, A. S. Bracker, and D. Gammon, Phys. Rev. B 73, 045307 (2006).

[22] X. Marie, T. Amand, P. Le Jeune, M. Paillard, P. Renucci, L. E. Golub, V. D. Dymnikov, and E. L. Ivchenko, Phys. Rev. B 60, 5811 (1999).

[23] J. Debus, D. Dunker, V. F. Sapega, D. R. Yakovlev, G. Karczewski, T. Wojtowicz, J. Kossut, and M. Bayer, Phys. Rev. B 87, 205316 (2013).

[24] C. Testelin, B. Eble, F. Bernardot, G. Karczewski, and M. Chamarro, Phys. Rev. B 77, 235306 (2008).

[25] C. Le Gall, A. Brunetti, H. Boukari, and L. Besombes, Phys. Rev. B 85, 195312 (2012). 